\title{
Méthode de préconcentration par extraction en phase solide : principe et application aux industries environnementales et pétrolières
}

\author{
F. Chapuis' ${ }^{1}$ V. Pichon ${ }^{1}$ et M.-C. Hennion' \\ 1 Laboratoire Environnement et Chimie analytique, École supérieure de Physique et Chimie industrielles de la Ville de Paris, \\ 10, rue Vauquelin, 75005 Paris - France \\ E-mail: florence.chapuis@espci.fr - valerie.pichon@espci.fr - marie-claire.hennion@espci.fr
}

\begin{abstract}
Résumé - Cet article a pour objectif de présenter les caractéristiques de l'extraction en phase solide pour les analyses environnementales à partir de matrices ou d'extraits liquides. Les principes fondamentaux qui régissent l'extraction sont expliqués afin de pouvoir choisir la nature et la quantité de phase extractante selon la matrice des échantillons et les propriétés des solutés recherchés en s'appuyant sur les mécanismes de rétention des phases. Les derniers développements concernant les formats disponibles, la nature des phases et l'automatisation sont présentés. Un intérêt particulier est porté aux phases sélectives émergentes, notamment celles qui agissent par reconnaissance moléculaire telles les immunoadsorbants ou les polymères à empreinte moléculaire permettant en une seule étape d'avoir un extrait propre. Les performances de leur couplage en ligne avec la séparation par chromatographie en phase liquide sont illustrées par des exemples dans le domaine environnemental. Quelques exemples particuliers à l'analyse des produits pétroliers sont également présentés.
\end{abstract}

\begin{abstract}
Preconcentration by Solid Phase Extraction: Principles and Applications in the Environmental and Petroleum Industries - The aim of this paper is to present the characteristics of solid phase extraction for environmental analysis using liquid matrices or extracts. The basic principles controlling the extraction are presented in order to aid in the choice of the nature and quantity of the extracting phase according to the sample matrix and the solute properties, based on the mechanisms of phase retention. Recent developments in available formats, types of phases and automation are considered. Special emphasis is placed on emerging selective phases, in particular those, such as immunoabsorbants molecularly imprinted polymers, which function via molecular recognition and allow selective extraction in a single step. Performance in on-line coupling with liquid phase chromatography is illustrated by examples from environmental analysis. Some examples specific to analysis of petroleum products are also presented.
\end{abstract}




\section{INTRODUCTION}

L'analyse environnementale est dans la majeure partie des cas une recherche de micropolluants présents à l'état de traces $(\mathrm{ng} / \mathrm{l}$ à $\mathrm{mg} / \mathrm{l})$ dans des échantillons à matrice parfois très complexe (rejets urbains et industriels, sols, boues de station d'épuration, etc.). Malgré les récents progrès de l'instrumentation analytique en termes de séparation et de détection, notamment avec la spectrométrie de masse, le recours à un traitement d'échantillon est nécessaire. Les analytes recherchés doivent être concentrés au préalable pour être détectables, surtout si la chromatographie en phase liquide (CPL) est utilisée comme méthode séparative. Cependant, dans les matrices complexes, on est confronté au problème de la coextraction d'autres analytes présents dans la matrice de l'échantillon et risquant d'interférer avec la méthode de séparation et de détection utilisée. L'élimination des interférents avant analyse est souvent une étape fastidieuse. À ce jour, on estime que le prétraitement des échantillons avant séparation chromatographique compte pour les deux tiers du temps total et constitue la première sources d'erreurs dans les résultats.

Aujourd'hui l'extraction en phase solide (SPE) est de loin la méthode la plus utilisée pour le traitement des échantillons liquides avant analyse [1]. C'est un domaine très actif des sciences séparatives et de nombreuses compagnies actuellement produisent et développent des nouveaux formats et phases d'extraction. Son émergence est particulièrement liée aux réglementations sur les solvants organiques mais aussi au fait que l'extraction liquide-liquide, longtemps utilisée, ne peut être appliquée à l'extraction de composés polaires qui ont peu d'affinité pour ces solvants. Son évolution a été facilitée par la commercialisation de phases très diverses dédiées à des composés et des matrices très variées soutenue par une évolution rapide des formats disponibles (cartouches de différentes capacités, disques, plaques à 96 puits), ainsi que des automates adaptés à ces différents formats.

La sélection d'un adsorbant conduisant, pour les analytes étudiés, à une forte rétention de ceux-ci est primordiale puisque, dans ce cas, il sera possible de percoler de grands volumes d'échantillons. Ceci est indispensable pour l'analyse des eaux pour laquelle les faibles niveaux de concentration recherchés impliquent l'obtention par l'étape d'extractionconcentration de facteurs d'enrichissement élevés. Pour l'extraction de composés contenus dans des échantillons aqueux, l'obtention d'une forte rétention implique l'utilisation de supports pour lesquels l'eau présente une faible force éluante. Il s'agit donc de supports hydrophobes comme les silices greffées par des chaînes hydrocarbonées de type $\mathrm{C}_{8}$ ou $\mathrm{C}_{18}$, des polymères de styrène divinylbenzène ou des phases de carbone. De par leur nature, ces supports induisent un mécanisme de rétention basé sur des interactions hydrophobes que les derniers développements tendent à amplifier notamment par augmentation de leur surface spécifique. Cependant, ce type d'interaction n'est pas sélectif et conduit aussi à une forte rétention de composés pouvant interférer au cours de l'analyse chromatographique. Malgré l'émergence de détecteurs sélectifs comme les spectromètres de masse, la quantification reste souvent délicate en raison de la présence de ces interférents. Il apparaît donc de plus en plus important de développer des méthodes d'extraction plus sélectives. Ceci peut se traduire par l'adjonction de procédures de purification, mais celles-ci peuvent être longues et l'ajout d'étape induit systématiquement une perte de temps et une augmentation de l'incertitude sur le résultat. Les dernières avancées en SPE se concrétisent alors par le développement de supports permettant un gain en sélectivité par l'association de plusieurs mécanismes comme celle du mécanisme de partage par interaction hydrophobe à l'échange d'ions pour les phases dites mixtes ou l'exclusion pour les phases à accès restreint. Ce gain en sélectivité est aussi la base du développement de phases stationnaires dont le mécanisme de rétention est lié à la reconnaissance structurale. Il s'agit, d'une part, d'immunoadsorbants, c'est-à-dire de supports greffés par des anticorps spécifiques capables de reconnaître des composés ayant une structure proche de celle du composé utilisé comme molécule de base pour l'immunisation, et d'autre part, de polymères à empreinte moléculaire dont la synthèse conduit à la création de cavité de la taille et de la forme de l'analyte recherché où siègent des interactions de nature variée et fonction de l'analyte.

Les paramètres qui guident le développement d'une procédure SPE sont résumés ci-dessous :

- la méthode séparative utilisée et la sensibilité de la détection. Ceci va dicter le volume d'échantillon nécessaire pour la préconcentration. Si la détection est très sélective, l'élimination des interférents n'est pas forcément nécessaire ;

- le nombre de composé à rechercher simultanément et leurs propriétés physico-chimiques (gamme de polarité, solubilité, etc.);

- La nature de l'échantillon et la quantité disponible ;

- Nombre d'échantillons à analyser (automatisation ou non, couplage en ligne extraction-séparation chromatographique) ;

- La réponse aux demandes actuelles : la diminution de l'emploi des solvants organiques, (surtout les solvants chlorés), le besoin d'analyses de plus en plus rapides et à moindre coût, le besoin de simplification de la procédure en limitant les interventions comme les évaporations ou transferts de solvants.

\section{PRINCIPE DE L'EXTRACTION EN PHASE SOLIDE ET SON ANALOGIE AVEC LA CHROMATOGRAPHIE EN PHASE LIQUIDE}

L'extraction sur phase solide est fondée sur la distribution des composés entre une phase solide (adsorbant) et une phase liquide (échantillon). 


\subsection{Procédure d'extraction}

Une procédure de l'extraction sur phase solide comporte trois ou quatre étapes en général. La première est le conditionnement de l'adsorbant contenu dans la cartouche d'extraction. Cette étape permet de mouiller le support en solvatant les groupements fonctionnels présents à sa surface. Ainsi un support hydrophobe est tout d'abord mouillé par un solvant organique, puis par un solvant de polarité similaire à celle du solvant constituant l'échantillon, généralement l'eau (ou un mélange eau-solvant organique). Lors de la seconde étape, on procède à la percolation de l'échantillon sur le support. Les interférents n'ayant aucune affinité avec la phase solide ne sont pas retenus sur le support lors de cette étape. Par contre, les molécules cibles et éventuellement des composés présentant une forte affinité avec l'adsorbant sont fixés sur le support. Selon la nature de l'échantillon (eaux, extraits de sol, etc.) et des composés à analyser et selon le facteur d'enrichissement recherché, le volume d'échantillon percolé peut varier de quelques microlitres jusqu'au litre pour la recherche de micropolluants organiques dans les eaux.

Une étape supplémentaire de lavage (étape 3) peut être effectuée de manière à éliminer les composés interférents faiblement retenus par le support. On choisira de ce fait un solvant de faible force éluante de façon à éluer les interférents tout en gardant fixés les composés d'intérêt.
Enfin, on procède à l'élution des composés ciblés en faisant percoler un solvant spécifiquement choisi pour rompre les interactions mises en jeu entre les analytes d'intérêt et le support solide en évitant, dans la mesure du possible, d'éluer des composés interférents fortement retenus sur le support.

\subsection{Analogie avec la chromatographie en phase liquide}

Par sa mise en œuvre décrite précédemment, il apparaît clairement que l'extraction sur phase solide est bien fondée sur un processus chromatographique, le support solide jouant le rôle de phase stationnaire et le solvant de l'échantillon puis l'éluant jouant successivement le rôle de phase mobile.

La mise en place d'un protocole d'extraction débute par l'étude des propriétés physico-chimiques des composés à analyser ainsi que la quantité et la nature de l'échantillon. Cette étude permet de choisir la nature chimique de l'adsorbant et la quantité de support conduisant à une forte affinité avec les analytes. Pour la recherche de micropolluants organiques tels que des pesticides dans des eaux de surface, il est important de définir, au préalable, le volume minimal à percoler pour atteindre une concentration suffisante d'analytes détectable par le système analytique. Cependant, lors de la percolation d'un grand volume d'échantillon sur la phase solide dans le but de concentrer au maximum les composés, a

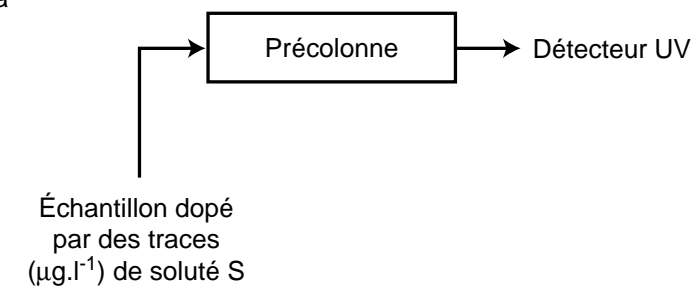

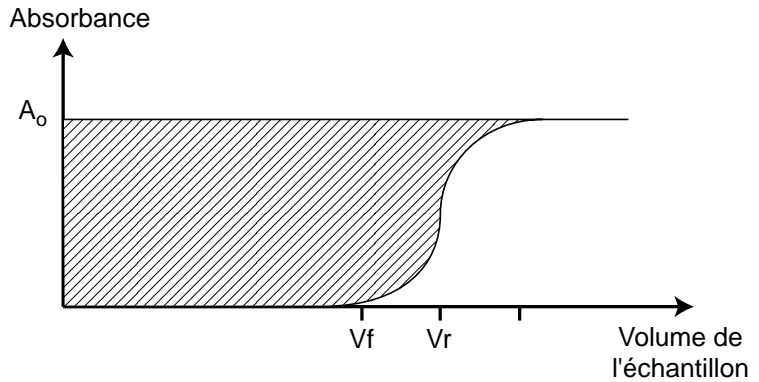

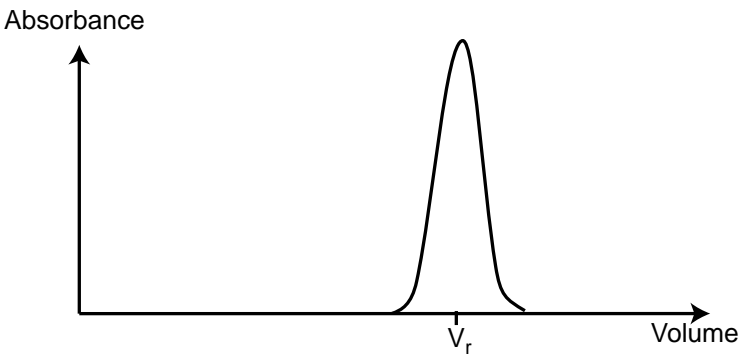

Figure 1

Détermination expérimentale du volume de rétention par chromatographie frontale (a) ou par chromatographie d'élution (b). Adapté de [2]. Experimental determination of retention volume by frontal chromatography (a) or by elution chromatography (b). Adapted from [2]. 
il est possible d'observer une perte de rétention de certains d'entre eux. En effet, pendant l'étape de percolation, le solvant constituant l'échantillon agit comme une phase éluante pour les solutés, si bien qu'à partir d'un certain volume percolé, appelé volume de fin de fixation, certains composés ne sont plus retenus quantitativement sur l'adsorbant.

Pour obtenir de bons rendements d'extraction, il est donc nécessaire de s'assurer de percoler un volume d'échantillon inférieur au volume de fin de fixation. Une connaissance préalable de ce volume est donc indispensable pour la mise en place d'un protocole d'extraction en particulier pour les échantillons environnementaux pour lesquels un facteur d'enrichissement élevé est recherché.

L'analogie de l'extraction sur phase solide avec un processus de chromatographie en phase liquide a été largement étudiée [1-5]. La figure 1a présente le front d'élution obtenu par percolation en continu d'un échantillon d'eau dopée par un soluté $\mathrm{S}$ sur un adsorbant en sortie duquel un dispositif de détection spectrophotométrie UV a été installé. Tant que le composé est retenu sur le support, l'absorbance mesurée est nulle. Par contre, sous l'effet éluant du solvant de percolation, le composé commence à être élué de la précolonne ce qui se traduit par une augmentation de l'absorbance. Le signal finit par atteindre une valeur constante $A_{0}$ qui correspond à l'absorbance de la concentration initiale du soluté dans la solution percolée, la précolonne ne retenant donc plus le soluté. Le front d'élution a idéalement l'allure d'une courbe bi-logarithmique correspondant à l'intégrale du pic d'élution obtenu par injection directe du soluté en chromatographie d'élution, incluant une colonne remplie par le même adsorbant et une phase mobile identique (fig. $1 b$ ). Le point d'inflexion de la courbe correspond au volume de rétention $V_{r}$ du soluté dans le solvant donné. Le volume de fin de fixation $V_{f}$ est défini pour un volume correspondant à $1 \%$ de $A_{0}$ l'absorbance initiale de l'échantillon.

La détermination des volumes de fin de fixation par analyse frontale est en fait peu utilisée car elle est difficile à mettre en œuvre expérimentalement. En effet, l'expérience est longue et la lecture du volume correspondant à $0,1 \%$ de $A_{0}$ souvent imprécise. Enfin, pour des composés ayant une très forte affinité avec le support, le front d'élution sera très étalé. Deux autres approches peuvent être envisagées. L'une d'entre elles consiste à préconcentrer des volumes croissants d'échantillon dopé toujours à quantité constante de composé. Tant que le $V_{f}$ n'est pas atteint, le composé est retenu lors de la percolation, on obtient alors des rendements d'extraction constants, égaux à $100 \%$ dans le cas idéal. Les rendements sont calculés en prenant, comme aire de référence, l'aire du pic d'élution obtenu après injection directe d'une quantité équivalente en chromatographie en phase liquide [6]. Après percolation d'un volume supérieur à $V_{f}$, le composé commence à être élué lors de la percolation de l'échantillon ce qui se traduit naturellement par une diminution de l'aire du pic chromatographique et par conséquent du rendement d'extraction. Une autre méthode s'appuie sur la détermination du facteur de rétention du soluté, noté $k$. En effet, en première approximation, le volume de fin de fixation peut être assimilé au volume de rétention $\left(V_{r}\right)$. Pour une phase stationnaire, un soluté et une phase mobile donnés, le volume de rétention et le facteur de rétention sont liés par l'équation suivante :

$$
V_{r}=(1+k) V_{0}
$$

$V_{0}$ est le volume mort de la précolonne et $k$, le facteur de rétention du soluté dans l'eau pure. Le volume mort peut se calculer à partir de la porosité et de la géométrie du support. Le facteur de rétention peut donc être déterminé par CPL en utilisant une colonne analytique remplie par le même adsorbant que celui que l'on souhaite utiliser en SPE. En ce qui concerne le facteur de rétention dans l'eau pure (en général trop élevé pour être mesurable directement), il peut être estimé à partir de la mesure des $\log k$ du soluté pour différentes proportions de solvant organique dans l'eau de la phase mobile. En effet, il existe une relation linéaire ou quadratique, suivant les conditions utilisées, entre le pourcentage de solvant organique introduit et la valeur de $\log k$. Ainsi, à partir de quelques mesures rapides de $\log k$ pour différents pourcentages de solvant organique, on obtient, par extrapolation, la valeur de $\log k$ dans l'eau pure $\left(k_{w}\right)$. Il s'agit d'une donnée approximative mais qui permet de prévoir rapidement le comportement d'un composé sur un support SPE.

\section{FORMATS ET AUTOMATISATION}

L'étape de préconcentration peut être totalement dissociée de l'analyse chromatographique (préconcentration dite «en différé ») et consiste alors en l'utilisation de cartouches (réservoirs remplis de particules de $40-60 \mu \mathrm{m}$ ) ou de disques imprégnés de phases jetables. Après percolation de l'échantillon sur l'adsorbant, les solutés piégés sont élués par passage d'un petit volume de solvant de force éluante élevée dont une partie est injectée dans le système chromatographique choisi. Cet extrait peut être réduit par évaporation avant injection de manière à gagner en facteur d'enrichissement. Cette étape peut également être intégrée au système chromatographique selon une préconcentration dite " en ligne ». Dans ce cas, la phase stationnaire est contenue dans une précolonne installée sur une vanne 6 voies à la place de la boucle d'injection : après préconcentration de l'échantillon par une première pompe (position de chargement), la phase mobile amenée par la pompe analytique assure ensuite le transfert des composés piégés vers la colonne analytique (position d'élution). Dans ce cas, la totalité des composés piégés sur l'adsorbant est transférée vers le système d'analyse. Il n'y a donc aucune perte en facteur d'enrichissement ce qui permet de traiter des volumes plus faibles que dans le cas de la méthode en différé pour laquelle seule une fraction de l'extrait est injectée et analysée. 
Les développements en SPE les plus récents concernent la commercialisation de phases sous de nombreux formats. Des phases sont disponibles en cartouche dans des réservoirs de tailles variées adaptées aux volumes d'échantillons à traiter. Des automates s'adaptant à tous les formats (cartouches remplies de particules ou de disques, plaques 96-puits, précolonnes pour les systèmes en ligne) permettent de traiter un grand nombre d'échantillons.

Outre la nature des phases qui reste le paramètre essentiel de la performance d'une méthode SPE, des supports particuliers ont été développés pour faciliter leur application à certaines matrices. En effet, si désormais les phases disponibles sont propres et directement utilisables, la qualité du polypropylène et polyéthylène utilisés pour les réservoirs et les frittés a été adaptée aux exigences de certains domaines d'application comme celui de la santé. Des systèmes de filtres ont été intégrés aux cartouches d'extraction de manière à éviter leur colmatage au cours de la percolation d'échantillons chargés en particules. Les disques se développent de plus en plus. Leur utilisation peut permettre de diminuer les volumes d'élution. Ils sont disponibles dans des réservoirs tout comme les phases à base de particules de façon à pouvoir être utilisés dans les automates. Ils se présentent aussi, chez certains fournisseurs, sous forme de disques de fibre de verre imprégnée de phases et sont donc plus fins et plus rigides que les membranes de PTFE et utilisables dans de simples dispositifs de filtration.

\section{CHOIX DE LA PHASE D'EXTRACTION}

Le choix de la phase d'extraction dépend d'abord de la matrice de l'échantillon. Si la phase est aqueuse, comme dans la majorité des échantillons biologiques et environnementaux, des supports hydrophobes conviennent parfaitement puisque l'eau est, sur ce type de phase, la phase mobile la moins éluante. Par contre, si l'échantillon est une phase organique, comme le sont les matrices huileuses ou à base de pétrole après dissolution dans un solvant apolaire comme l'isooctane, les extractants retenus seront polaires, tels les silices vierges ou greffées polaires, ou les alumines.

Dans un deuxième temps, le choix de la phase va dépendre des caractéristiques physico-chimiques des solutés recherchés et notamment de leur polarité. La polarité d'un soluté est difficile à définir, mais elle peut être toutefois reliée à la notion d'hydrophobie définie à partir de la constante de partage octanol-eau $\left(\mathrm{P}_{\mathrm{oct}}\right)$. Ainsi, sont considérés comme modérément polaires des composés dont le logarithme de $\mathrm{P}_{\text {oct }}$ est compris entre 1 et 3 , comme polaires ceux dont le logarithme de $\mathrm{P}_{\text {oct }}$ est inférieur à 1 et comme apolaires ceux dont le logarithme de $\mathrm{P}_{\text {oct }}$ est supérieure à 3 . C'est en fonction de ce classement que peut s'effectuer la sélection des supports.

\subsection{Phases hydrophobes}

\subsubsection{Silices greffées}

Déjà largement utilisées comme phases stationnaires en CPL, les silices greffées $\mathrm{C}_{8}$ ou $\mathrm{C}_{18}$ ont été longtemps les phases les plus utilisées en SPE pour le traitement d'échantillons aqueux. Leur évolution a été menée avec des objectifs différents de ceux de la CPL à savoir favoriser les interactions hydrophobes par augmentation de la surface spécifique jusqu'à $500-600 \mathrm{~m}^{2} / \mathrm{g}$. Pour les silices greffées $\mathrm{C}_{18}$, on peut facilement estimer le volume de fin de fixation à partir de la constante de partage octanol-eau, puisque la valeur de $\log k$ dans l'eau pure $\left(k_{w}\right)$ des solutés est du même ordre de grandeur, comme le montre le tableau 1.

\section{TABLEAU 1}

Volumes de rétention calculés $V_{r}$ pour $100 \mathrm{mg}$ de silice greffée $\mathrm{C}_{18}$ à partir des valeurs extrapolées log $\mathrm{k}$ dans l'eau pure. Adapté de [2].

Retention volumes $V_{r}$ for $100 \mathrm{mg}$ of $C_{18}$ grafted silica, calculated by extrapolation of values for log $k$ for pure water. Adapted from [2].

\begin{tabular}{l|c|c|c}
\hline Composés & Log $\mathrm{P}_{\text {oct }}$ & $\log k$ & $\begin{array}{c}V_{r}, 100 \mathrm{mg} \\
\text { de support }\end{array}$ \\
\hline pentachlorophénol & 5 & 4,8 & 7350 \\
2, 4, 5-trichlorophénol & 4,1 & 4,0 & 1064 \\
3, 5-trichlorophénol & 3,56 & 3,5 & 370 \\
2, 4-dichlorophénol & 3,2 & 3,1 & 160 \\
4-chlorophénol & 2,4 & 2,3 & 32 \\
phénol & 1,49 & 1,55 & 4,4 \\
\hline
\end{tabular}

Ainsi, on peut voir la limitation des silices $\mathrm{C}_{18}$ pour l'extraction de composés polaires comme le phénol, puisque avec $100 \mathrm{mg}$ de phase le rendement sera inférieur à $100 \%$ dès que le volume dépassera $5 \mathrm{ml}$.

Parallèlement, si la suppression des silanols résiduels pour les phases de CPL peut avoir son importance sur la qualité des séparations, la présence de ces fonctions peut conduire en SPE à des interactions additionnelles et favoriser l'extraction de composés polaires, les interactions hydrophobes restant cependant prépondérantes dans le mécanisme de rétention. De ce fait, l'application de ces supports se limite généralement aux composés peu polaires, caractérisés par des valeurs de $\log \mathrm{P}_{\mathrm{oct}}$ supérieures à 2 surtout lorsqu'il est nécessaire de percoler de grands volumes d'échantillons comme c'est le cas pour l'analyse des eaux.

Pour des composés fortement hydrophobes pour lesquels ces supports conviennent, il peut être intéressant d'optimiser le choix du greffage en optant par exemple pour des chaînes hydrocarbonées courtes ce qui facilite leur élution ou des phases greffées par des fonctions cyclohexyle ou phényle qui sont adaptées à l'extraction de composés possédant des noyaux aromatiques. 


\subsubsection{Polymères de polystyrène-divinylbenzène (PS-DVB)}

L'extraction de composés plus polaires se fait préférentiellement sur des supports à base de polymères. Les premiers polymères développés comme phases de SPE ont été des supports utilisés en CPL. Il s'agit des copolymères apolaires de polystyrène-divinylbenzène de PLRP-S (Polymer Laboratories) et de PRP-1 (Hamilton) de surface spécifique de 550 et $415 \mathrm{~m}^{2} / \mathrm{g}$ respectivement. Ces phases ne sont disponibles qu'en format de précolonne à utiliser en ligne. Des supports similaires disponibles en cartouches ou sous forme de disques d'extraction ont ensuite été commercialisés. De nombreuses études ont montré l'intérêt de ces supports par rapport aux phases de silice greffée $\mathrm{C}_{18}$ permettant, de par leur plus grand pouvoir hydrophobe, d'augmenter d'un facteur 10 à 40 les volumes percolables sans perte en rendement, surtout pour les composés à noyaux aromatiques sujets aux interactions de type $\pi-\pi$ [7]. De plus, contrairement aux phases de silice greffée, ces phases peuvent être utilisées sur toute la gamme de $\mathrm{pH}$ entre 0 et 14 . Cependant, pour augmenter la rétention et extraire des composés de plus en plus polaires, des supports de plus haute surface spécifique ont été développés plus récemment. Ainsi pour un composé polaire comme la deisopropylatrazine $\left(\log \mathrm{P}_{\mathrm{oct}}=1,2\right)$ le rendement d'extraction est d'environ $20 \%$ en percolant $500 \mathrm{ml}$ d'échantillon sur un cartouche remplie de $500 \mathrm{mg}$ de silice $\mathrm{C}_{18}$, alors que le volume de fixation est supérieur à 1 litre en utilisant une cartouche remplie de $200 \mathrm{mg}$ de PS-DVB ayant une surface spécifique de $1000 \mathrm{~m}^{2} / \mathrm{g}$. De nombreuses études menées dans le domaine environnemental ont montré l'intérêt de disposer de grandes surfaces spécifiques avec notamment des applications aux métabolites polaires des triazines [5] et aux phénols [8]. Des polymères fonctionnalisés par des groupements polaires ont aussi été synthétisés de manière à améliorer le contact avec l'échantillon aqueux pendant la percolation. Parallèlement, des phases basées sur une copolymérisation de divinylbenzène avec un monomère polaire (comme le N-vinylpyrolidone pour la phase Oasis HLB) ont aussi été développées pour améliorer la mouillabilité des phases et favoriser les interactions avec les composés polaires. Des polymères modifiés par des groupements ioniques sont aussi disponibles et engendrent des mécanismes différents (voir phases mixtes).

\subsubsection{Carbone graphite poreux (PGC)}

Le support carboné le plus utilisé est le GCB (Graphitized Carbon Black, noir de carbone). Il est caractérisé par une structure homogène bien ordonnée de surface spécifique de l'ordre de $120 \mathrm{~m}^{2} / \mathrm{g}$. Malgré cette faible surface spécifique, sa plus grande efficacité que la silice greffée $\mathrm{C}_{18}$ à piéger les composés polaires a été largement démontrée tout comme la possibilité d'utiliser des charges résiduelles positives pour réaliser des extractions fractionnées de composés acides d'une part, neutres et basiques d'autre part [9]. Un autre sup- port de carbone caractérisé par une structure cristalline en feuillet de plans d'atomes de carbone, le PGC (Porous Graphitic Carbon, carbone graphite poreux) a lui aussi été très utilisé pour l'extraction de composés polaires. Sur ce support, les composés sont retenus par interactions hydrophobes mais aussi électroniques permettant d'extraire aussi bien des composés apolaires que des composés polaires très solubles dans l'eau [10-12].

Des facteurs de rétention dans l'eau pure pour la simazine et différents métabolites polaires des triazines ont été déterminés pour une colonne de silice greffée $\mathrm{C}_{18}$. Ils sont comparés aux facteurs de rétention obtenus sur une phase polymérique de PS-DVB (PRP-1, Hamilton) et pour une colonne de carbone graphitisé poreux (PGC, Hypersil). Ces valeurs sont répertoriées dans le tableau 2, les composés sont classés suivant leur caractère hydrophobe.

\section{TABLEAU 2}

Facteurs de rétention $(\log k)$ mesurés dans l'eau pure ou obtenus par extrapolation sur différents supports [5].

Retention factors ( $\log k$ ) for different supports measured in pure water or obtained by extrapolation [5].

\begin{tabular}{l|c|c|c|c}
\hline Composés & $\log \mathrm{P} \mathrm{C}_{18}$ & $\log k$ & $\log k$ PRP-1 & $\log k$ PGC \\
\hline Amméline & $-1,2$ & $<0,5$ & $<0,5$ & $2,4 \pm 0,2$ \\
Ammélide & $-0,7$ & $<0,5$ & $<0,5$ & $2,5 \pm 0,2$ \\
OH DIA & $-0,1$ & $1,0 \pm 0,1$ & $1,0 \pm 0,1$ & $3,0 \pm 0,2$ \\
OH DEA & 0,2 & $1,5 \pm 0,1$ & $1,8 \pm 0,1$ & $2,8 \pm 0,2$ \\
DIA & 1,2 & $2,3 \pm 0,1$ & $3,1 \pm 0,1$ & $>3,5$ \\
DEA & 1,4 & $2,7 \pm 0,1$ & $3,5 \pm 0,3$ & $3,2 \pm 0,2$ \\
Simazine & 2,3 & $3,4 \pm 0,2$ & $>4$ & $>4$ \\
\hline
\end{tabular}

La rétention des composés polaires est tellement forte qu'il apparaît dans ce cas impossible d'utiliser ce support en ligne avec une colonne analytique de silice greffée $\mathrm{C}_{18}$. En effet, la rétention sur silice greffée $\mathrm{C}_{18}$ implique pour ces composés polaires une phase mobile riche en eau qui, de ce fait, n'est pas suffisamment éluante pour transférer les solutés de la précolonne de PGC sur la colonne analytique. Le même problème peut être rencontré lorsque l'on utilise des copolymères de très haute surface spécifique. Toutefois il peut être contourné par l'utilisation d'une colonne analytique de PGC pour la séparation qui est alors réalisée avec une phase mobile plus riche en solvant organique [13]. Il est aussi possible d'utiliser une pompe analytique permettant de désorber les solutés de la phase carbonée par le solvant organique pur auquel est ajoutée, après la précolonne et avant la colonne analytique et ce par une pièce en $\mathrm{T}$, la quantité d'eau nécessaire pour constituer la phase mobile adaptée à la séparation sur silice greffée [14]. Diverses applications des phases carbonées à l'extraction de composés polaires de matrices aqueuses sont présentées dans la référence [12]. 


\subsubsection{Limitation des supports hydrophobes}

La classification des composés en fonction de leur hydrophobie permet de faire un choix raisonné des phases à utiliser. Quand l'étape d'extraction doit s'adapter à des composés appartenant à une large gamme de polarité, il peut s'avérer nécessaire de procéder à deux extractions distinctes : une méthode pour les composés les plus polaires sur des phases de polymères à haute surface spécifique ou de carbone ; les composés apolaires étant traités par extraction sur silice greffée avec l'ajout éventuel d'une faible proportion de solvant organique pour limiter les pertes dues à l'adsorption des composés très hydrophobes sur les parois des matériels utilisés [15]. Cependant, si l'on arrive dans bien des cas à adapter le choix des phases aux composés à extraire, l'application seule de ces supports à des matrices complexes, comme les fluides biologiques ou des eaux fortement contaminées, conduit à la co-extraction par interactions de nature hydrophobe d'autres composés présents dans l'échantillon qui peuvent nuire à la détection et la quantification des analytes recherchés. De nombreuses méthodes de purification des extraits sur des supports polaires (silice, alumine, Florisil, etc.) ont été décrites dans la littérature. Elles permettent de limiter l'injection dans le système chromatographique d'une grande partie des interférents. Cependant, le mode de rétention/élution étant encore lié, sur ces supports, à la polarité des composés, ces étapes longues à mettre en œuvre ne sont pas toujours suffisamment performantes. Il apparaît donc de plus en plus important d'utiliser des supports apportant davantage de sélectivité dans les mécanismes de rétention.

\subsection{Phases sélectives}

\subsection{1 Échangeurs d'ions et phases mixtes}

Dans le cas de composés ioniques ou ionisables, les interactions mises en jeu lors de l'extraction peuvent être de nature ionique en adaptant le $\mathrm{pH}$ de la solution. Le mécanisme de rétention est alors fondé sur un mécanisme d'échange d'ions. De la silice ou des résines polymériques sont greffées par des groupements acides (acides carboxylique ou sulfonique) ou basiques (amine) pour jouer le rôle respectivement d'échangeur de cations et d'échangeur d'anions. Le principe de l'extraction consiste alors à retenir les composés par interactions électrostatiques sous leur forme ionisée et à les éluer en se plaçant à un $\mathrm{pH}$ pour lequel ils sont sous la forme moléculaire. Dans l'impossibilité de se placer dans une zone où le composé est neutre, l'élution peut être réalisée par une solution de grande force ionique. Cependant, ce mécanisme de rétention est rarement mis en œuvre pour l'analyse directe de traces de composés dans les échantillons complexes car ces derniers contiennent de trop fortes teneurs en ions inorganiques et saturent la capacité de l'échangeur.
Il a été montré que les silanols résiduels ionisés présents en quantité non négligeable suivant le pH de l'échantillon sur les phases de silice greffée par des chaînes alkyles facilitaient l'extraction de composés basiques azotés. Pour augmenter ces interactions électrostatiques, des phases ont été greffées à la fois par des chaînes alkyles et des fonctions ionisées de type acide fort $\left(\mathrm{SO}_{3}{ }^{-}\right)$et bases fortes $\left(\mathrm{NR}_{3}{ }^{+}\right)$. Le soluté peut alors être retenu par interactions électrostatiques fortes et par interactions hydrophobes. Au cours de la percolation de l'échantillon, le soluté est piégé par interaction hydrophobe sur le support alors que les composés polaires non chargés ou portant une charge identique à celle de l'échangeur d'ions greffé ne sont pas retenus. On percole alors une solution aqueuse portée à un $\mathrm{pH}$ permettant l'ionisation du soluté qui développe alors des interactions électrostatiques avec l'échangeur d'ions. Ces interactions étant de forte énergie, il est alors possible de procéder à un lavage par un solvant organique dans lequel a été ajouté un acide ou une base pour maintenir l'ionisation du soluté. Le solvant organique agissant sur les interactions hydrophobes, les composés uniquement retenus par les chaînes alkyles sont alors éliminés. Après cette étape de lavage, le soluté est élué par passage de ce solvant organique ajusté à une valeur de $\mathrm{pH}$ ne permettant plus son ionisation afin de rompre les interactions électrostatiques.

\subsubsection{Phases à accès restreint (RAM)}

Ces dernières années, des phases dites à accès restreint (Restricted Acces Media, RAM) ont été développées afin de fractionner l'échantillon et permettre l'injection directe de matrices biologiques dans un système en ligne avec la CPL. Ces phases combinent un mécanisme d'exclusion des molécules de haut poids moléculaire et un mécanisme de partage permettant la rétention des petites molécules. De nombreuses phases ont été développées [16,17]. L'une des plus utilisées, nommée ISRP (Internal Surface Reversed Phase), permet de combiner un mécanisme d'exclusion et un mécanisme de rétention par interactions hydrophobes. Une barrière physique liée à une taille de pore limitée empêche l'accès des molécules de grandes tailles comme les protéines à la surface interne de la phase stationnaire poreuse. De plus, un traitement spécifique permet de rendre hydrophile la surface externe de la phase et donc de limiter l'adsorption des protéines. Enfin, la présence de chaînes hydrophobes greffées sur la surface poreuse interne des grains permet la rétention des solutés à extraire. Ces phases sont utilisées pour éliminer l'influence des acides humiques dans les eaux de surface par exemple.

\subsubsection{Immunoadsorbants}

Pour augmenter la sélectivité de l'étape d'extraction, des supports mettant en œuvre des anticorps spécifiques d'une molécule et greffés sur un support solide (silice, gel d'agarose) ont été développés. 
L'emploi d'anticorps comme réactif analytique n'est pas récent dans le domaine médical et biologique, à la différence du domaine environnemental pour la recherche de micropolluants. Une des raisons réside dans la difficulté de produire des anticorps très spécifiques de molécules de petite taille. Les mammifères ne développant pas de réponse immunitaire envers ces molécules, il est nécessaire de coupler la molécule-antigène choisie à une protéine porteuse ce qui impose souvent de la modifier au préalable par l'introduction d'un groupement fonctionnel.

Dans le cas des molécules de petite taille et qui possèdent de ce fait peu de groupements caractéristiques, la probabilité pour que les anticorps ainsi produits reconnaissent d'autres composés de structure voisine est assez grande. C'est ce qu'on nomme la réactivité croisée des anticorps. Cette propriété est un avantage puisque, dans le cas de la recherche de polluants, on est souvent amené à rechercher de nombreux composés d'une même famille en incluant éventuellement des métabolites. Les développements et l'utilisation de ces supports ont fait l'objet de nombreuses revues [18-22].

La première étape de synthèse d'un immunoadsorbant consiste en la production des anticorps. Pour cela, il faut sélectionner un composé représentatif de la famille ciblée, l'antigène, qui sera pris comme modèle pour produire les anticorps. Cet analyte doit souvent être chimiquement modifié afin d'introduire une fonction qui permettra son greffage à une protéine porteuse. Plusieurs molécules modifiées, appelées haptènes, seront couplées à une protéine. Cette entité immunogène, appelée immunoconjugué, est ensuite utilisée pour immuniser le mammifère. Quelques $\mathrm{ml}$ de sérum sont ensuite prélevés de l'animal après quelques semaines d'immunisation. Une purification est mise en œuvre pour isoler les anticorps (immunoglobulines de type $\mathrm{G}, \mathrm{IgG}$ ) et les greffer chimiquement sur un support solide hydrophile afin qu'il ne génère pas d'interactions pouvant conduire à terme à une rétention non spécifique sur l'immunoadsorbant. Dans une première approche, les immunoadsorbants (IS) sont synthétisés avec des anticorps polyclonaux, à savoir la fraction d'immunoglobulines de type $\mathrm{G}(\mathrm{IgG})$ que l'on isole du sérum. Cette fraction contient des anticorps d'affinité variées et seuls 10 à $15 \%$ d'entre eux sont spécifiques de l'immunoconjugué et donc de la molécule ciblée. Ces anticorps sont moins chers et plus rapides à produire que les anticorps monoclonaux qui sont issus de la sélection du clone générant les anticorps de spécificité souhaitée. Ces derniers sont cependant préconisés pour la commercialisation des IS car, une fois la lignée sélectionnée, ils sont obtenus de façon reproductible, en grande quantité et sans recourir à l'utilisation d'animaux.

Les immunoadsorbants ainsi produits peuvent être introduits dans des réservoirs de type cartouches ou précolonnes entre deux frittés et être utilisés comme tout support classique d'extraction : après une étape de conditionnement par un tampon et de l'eau pure, l'échantillon aqueux est appliqué, un lavage à l'eau pure est alors éventuellement introduit.
L'application d'un solvant d'élution permet de récupérer les solutés sélectivement retenus par les anticorps.

Les propriétés des immunoadsorbants résultent surtout de celles des anticorps greffés. Si les anticorps créent des interactions spécifiques avec un antigène donné contre lequel ils ont été synthétisés, ils sont aussi source de réactions croisées, c'est-à-dire de réactions avec les analogues structuraux de l'antigène. En principe, une forte affinité des anticorps est obtenue pour la molécule utilisée pour la synthèse des anticorps (molécule antigène). L'affinité pour les autres molécules de la même famille chimique va dépendre des caractéristiques structurales des molécules. Cette affinité va influer sur la rétention des composés au cours de l'étape d'extraction et donc sur les rendements d'extraction pour un volume d'échantillon donné. Par modélisation moléculaire des triazines et des immunoconjugués et par l'exploitation des résultats par analyse en composante principale, il a récemment été mis en évidence l'importance du volume des groupements caractérisant les différentes molécules et des interactions électroniques (mesurées par la répartition des charges dans les molécules) dans le mécanisme de reconnaissance par les anticorps et donc leur affinité vis à vis des différents composés de cette famille [23].

Un rendement d'extraction inférieur à $100 \%$ peut être dû à une faible affinité des anticorps vis-à-vis du composé étudié mais aussi à un dépassement de capacité. Il est donc important de connaître ce paramètre couramment défini comme le nombre total de sites de reconnaissance disponibles sur l'IS et capables de fixer les analytes. Cette capacité est donc directement liée au nombre et à la nature des anticorps greffés ainsi qu'à leur accessibilité et peut être facilement mesurée par percolation d'un volume constant d'eau dopée par des quantités croissantes d'analyte-antigène.

Le protocole d'extraction sur immunoadsorbants est similaire à celui utilisé pour les supports classiques d'extraction (silice greffée $\mathrm{C}_{18}$, copolymères apolaires, etc.). Après conditionnement de l'immunoadsorbant par quelques millilitres d'eau pure, l'échantillon est percolé. Les solutés sélectivement retenus par les anticorps sont ensuite désorbés par un faible volume d'éluant. Si les grosses molécules (protéines, hormones, etc.) sont désorbées par simple changement de $\mathrm{pH}$, modification de la force ionique de l'éluant ou ajout d'agents dénaturants, ces conditions sont peu efficaces pour la désorption de petites molécules. Seuls des solvants organiques comme le méthanol ou l'acétonitrile, permettent une désorption efficace de l'analyte en un faible volume et des mélanges hydro-organiques contenant jusqu'à $80 \%$ de méthanol ou d'acétonitrile peuvent être utilisés sans endommager l'immunoadsorbant à la différence d'anticorps non greffés dont la dénaturation est irréversible dans de telles conditions. En effet, l'IS est régénéré par stockage à $4{ }^{\circ} \mathrm{C}$ dans une solution aqueuse tamponnée contenant un agent anti-bactérien. 


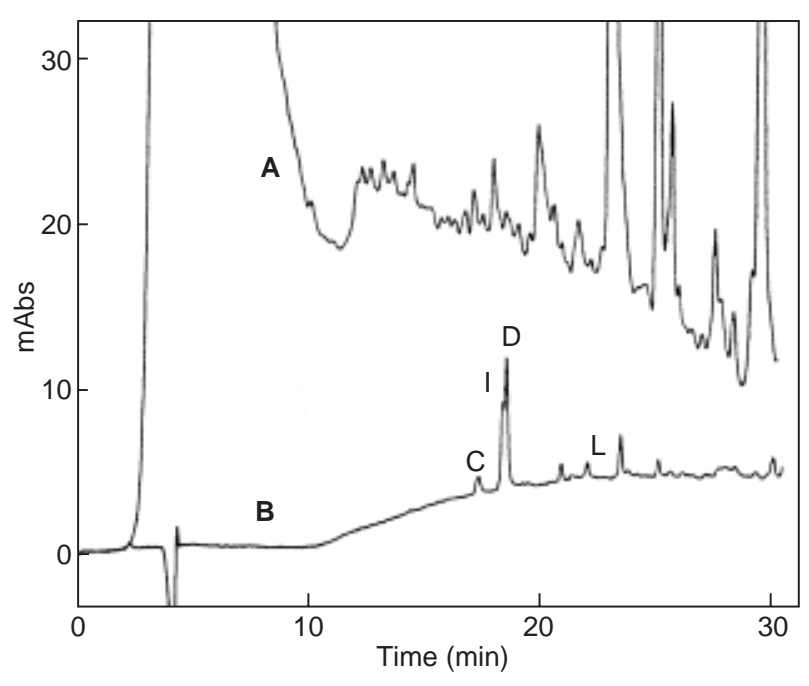

Figure 2

Couplage en ligne de silice greffée $\mathrm{C}_{18}$ (A) et d'un immunoadsorbant anti-phénylurées (B) avec la $\mu \mathrm{CPL} / \mathrm{UV}$ pour l'analyse de $5 \mathrm{ml}$ d'eau de Seine. Détection UV à $244 \mathrm{~nm}$ de Chlortoluron (C, $50 \mathrm{ng} / \mathrm{l}$ ), Isoproturon (I, $220 \mathrm{ng} / \mathrm{l}$ ), Diuron $(\mathrm{D}, 200 \mathrm{ng} / \mathrm{l})$ et Linuron (L, $40 \mathrm{ng} / \mathrm{l})$. D'après [31].

On-line coupling of $C_{18}$ silica (A) and an antiphenylurea immunosorbent (B) using $\mu C P L / U V$ for the analysis of $5 \mathrm{ml}$ of water from the Seine. UV detection at $244 \mathrm{~nm}$ of Chlortoluron (C, $50 \mathrm{ng} / \mathrm{l})$, Isoproturon (I, $220 \mathrm{ng} / \mathrm{l})$, Diuron (D, $200 \mathrm{ng} / \mathrm{l})$ and Linuron $(L, 40 \mathrm{ng} / \mathrm{l})$. From [31].

Généralement conditionnés dans des cartouches d'extraction en polypropylène, piégés entre deux frittés, les immunoadsorbants peuvent également être introduits dans des précolonnes intégrées dans des montages permettant de coupler en ligne l'extraction et l'analyse par CPL. La désorption est alors réalisée par le gradient de phase mobile utilisé pour la séparation analytique. Cette phase mobile doit contenir une quantité suffisante de solvants organiques pour permettre la désorption des solutés tout en permettant la séparation des composés ciblés. Des anticorps greffés sur silice ont été utilisés pour l'analyse en ligne de triazines, de phénylurées, de HAP et de colorants dans des eaux [24-33]. Dans ces différents cas, une phase mobile contenant de l'eau et de l'acétonitrile a permis un transfert rapide des solutés vers la colonne analytique sans perte en efficacité.

Un exemple illustrant bien la grande sélectivité des immunoadsorbants est donné sur la Figure 2 correspondant à l'analyse en ligne de $5 \mathrm{ml}$ d'eau de Seine contaminée par des phénylurées [31]. Le chromatogramme de la Figure 2a, correspondant à la préconcentration de cet échantillon sur silice greffée $\mathrm{C}_{18}$, est marqué par la présence de nombreux interférents aux mêmes temps de rétention que les phénylurées recherchées. Ces pesticides sont en revanche facilement quantifiables après immuno-préconcentration (Fig. $2 b$ ) puisque aucun autre composé n'a été co-extrait par les anticorps anti-phénylurées utilisés pour cette étude. Un autre exemple est représenté Figure 3, avec l'analyse en ligne des hydrocarbures polyaromatiques dans les eaux de surface, montrant qu'à partir de $80 \mathrm{ml}$ d'eau on peut detecter ces composés avec des limites de détection inférieures à $10 \mathrm{ng} / \mathrm{l}$ par UV, avec confirmation par le spectre UV, très informatif pour ces composés, en plus de la détection par fluorescence [30].

\subsubsection{Polymères à empreinte moléculaire (MIP)}

La grande sélectivité apportée par les immunoadsorbants a conduit à la synthèse de polymères à empreinte moléculaire (MIP) basés sur la formation, dans le polymère, de cavités mimant les sites de reconnaissance spécifique obtenus avec les anticorps. Outre le fait d'être peu onéreux si la molécule modèle est disponible à faible coût, de tels polymères présentent une excellente stabilité chimique, mécanique et thermique. Les ouvrages ou revues générales portant sur le sujet [34-36] et le nombre de publications en augmentation exponentielle durant ces cinq dernières années témoignent du grand potentiel des MIP pour l'extraction de polluants de matrices variées.

Ils sont obtenus par polymérisation. La première étape de la synthèse consiste à mettre en contact la molécule modèle, le(s) monomère(s) fonctionnel(s) et un agent réticulant dans un solvant porogène qui va permettre de créer une structure meso- ou macroporeuse. Le monomère est choisi de manière à développer, avec la molécule empreinte, des liaisons covalentes, non covalentes ou encore des liaisons de coordination. Cependant, la plus grande simplicité de mise en œuvre de liaisons non covalentes fait que cette méthode est plus largement représentée dans la littérature pour l'application des MIP comme support d'extraction. Cette méthode permet un bon compromis entre une interaction analyte-monomères suffisamment forte pour l'obtention d'une grande sélectivité et une désorption rapide ce qui est primordial pour un éventuel couplage en ligne des polymères à la CPL. Les polymères sont dans la plupart des cas synthétisés en bloc puis broyés et tamisés de manière à obtenir des particules de taille homogène (comprise entre 25 et $50 \mu \mathrm{m}$ ) que l'on conditionne ensuite sous forme de cartouche ou de colonne.

Le choix de la molécule modèle est important. De la même manière que l'haptène et donc l'immunoconjugué définissent la spécificité des anticorps produits, la structure de la molécule empreinte, qui possède une géométrie et des fonctionnalités particulières, va définir la spécificité du MIP et on peut obtenir des MIP capables de reconnaître une famille de composés comme les triazines [37]. Après synthèse, il est important d'éliminer totalement cette molécule empreinte pour qu'elle ne soit pas relarguée par le polymère au cours de son utilisation conduisant ainsi à des faux positifs. Cette élimination totale est parfois incertaine $[38,39]$ et conduit certains groupes à utiliser, comme empreinte pour la 


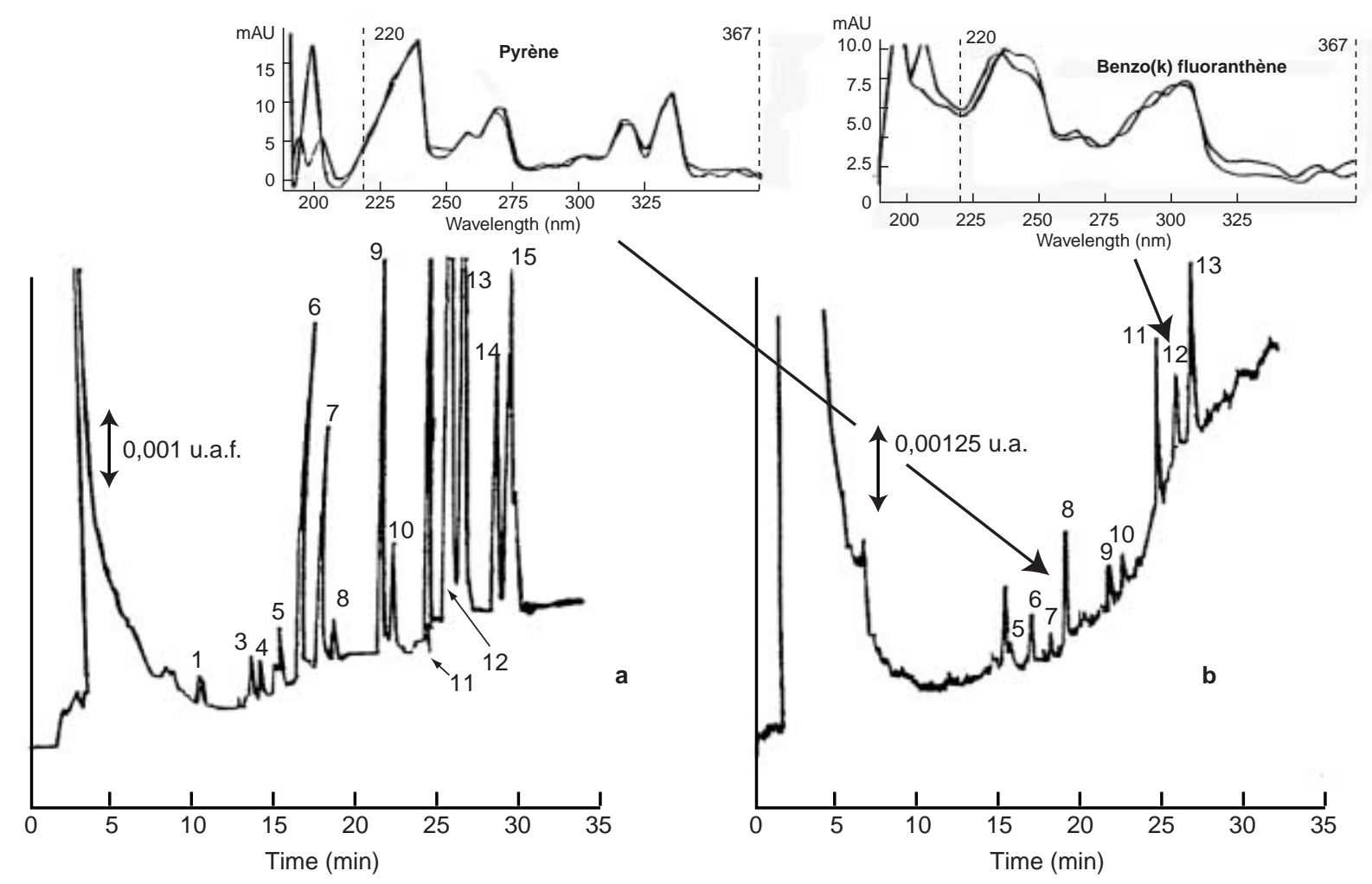

Figure 3

Couplage en ligne d'un immunoadsorbant anti-fluorène avec la CPL pour l'analyse des hydrocarbures aromatiques dans les eaux de surface (80 ml d'eau de Seine) contenant $10 \%$ d'acétonitrile, dopée avec $20 \mathrm{ng} / 1$ de chaque composé (1 - naphthalène ; 3 - acénaphthène ; 4 - fluorène ; 5 - phenanthrène ; 6 - anthracène ; 7 - fluoranthène ; 8 -pyrène ; 9 - benzo(a)anthracène ; 10 - chrysène ; 11 - benzo(b)fluoranthène ; 12 - benzo(k)fluoranthène ; 13 - benzo(b)pyrène ; 14 - dibenzo(ah)anthracène ; 15 - benzo(ghi)perylène). Détection par fluorescence (a) et par UV à $239 \mathrm{~nm}$ (b) avec en encart la confirmation par les spectres UV de deux composés. D'après [30].

On-line coupling of an antifluorene immunoadsorbant with LC for the analysis of aromatic hydrocarbons in surface waters $(80 \mathrm{ml}$ of water from the Seine) containing 10\% acetonitrile, spiked with $20 \mathrm{ng} / \mathrm{l}$ of each component (1 - naphthalene; 3 - acenaphthene; 4 - fluorene; 5 - phenanthrene; 6 - anthracene; 7 - fluoranthene; 8 - pyrene; 9 - benzo(a)anthracene; 10 - chrysene; 11 - benzo(b)fluoranthene; 12 - benzo(k)fluoranthene; 13 - benzo(b)pyrene; 14 - dibenzo(ah)anthracene; 15 - benzo(ghi)perylene). Detection by fluorescence (a) and by UV at $239 \mathrm{~nm}$ (b). Insert shows UV spectroscopy confirmation of two compounds. From[30].

synthèse, un analogue structural de la molécule cible qui n'est pas susceptible d'être présent dans les échantillons.

Le monomère est choisi pour sa capacité à établir des interactions fortes non covalentes avec la molécule empreinte dans le solvant de polymérisation. La majorité des MIP utilisés en extraction ont été synthétisés à partir de l'acide méthacrylique. Ce monomère est susceptible de développer, suivant les molécules empreintes, des interactions de type liaison hydrogène ou des interactions électrostatiques. Des monomères basiques tels que la 4-vinylpyridine pouvant générer des interactions de type $\pi$ - $\pi$ avec certains analytes peuvent également être utilisés.

La majorité des réaction de polymérisation décrites utilise, comme agent réticulant, le diméthacrylate d'éthylène glycol, ce qui s'explique notamment par sa grande stabilité mécanique et thermique.
Le solvant de polymérisation est un paramètre-clé de la spécificité du MIP puisqu'il conditionne la nature et la force des interactions entre la molécule empreinte et le(s) monomère(s). La spécificité des cavités du MIP dépend de la stabilité du complexe formé. Les solvants les plus utilisés sont des solvants peu polaires et aprotiques de façon à favoriser les interactions préalablement citées comme les liaisons hydrogène. La nature du solvant de polymérisation influe aussi sur la structure et la morphologie du polymère en modifiant sa porosité (effet porogène) et sa capacité à gonfler en présence de certains solvants utilisés au cours de l'étape d'extraction.

Pour qu'un analyte soit extrait spécifiquement par le MIP, il faut que sa structure soit relativement proche de celle de l'empreinte pour pénétrer dans les cavités et ensuite interagir avec le polymère. L'analyte cible sera retenu spécifiquement dans les cavités du polymère s'il développe le même type 
d'interactions que celles développées lors de la synthèse. Cela implique donc l'utilisation pour l'extraction sur MIP d'un solvant analogue au solvant de synthèse. Le solvant de polymérisation étant de nature organique, l'extraction de composés d'échantillons aqueux implique une première étape de transfert dans un solvant organique par extraction liquideliquide [40] ou par extraction sur un support hydrophobe conventionnel [41-43]. Un exemple d'application est donné Figure 4. Il correspond à l'analyse d'une eau de source dopée par des triazines et leurs métabolites $(100 \mathrm{ml}$ à $0.5 \mu \mathrm{g} / \mathrm{l})$ après extraction sur un polymère hydrophobe (polystyrène divinylbenzène) suivie (a) ou non d'une étape de purification sur un MIP (b). La comparaison des deux chromatogrammes illustre le gain en sélectivité puisque le passage sur le MIP a permis d'éliminer une grande quantité de composés préalablement extraits par le polymère hydrophobe.

L'échantillon aqueux peut être directement percolé sur le MIP mais une étape de lavage par le solvant qui permet de générer les interactions spécifiques au sein des cavités est alors obligatoire. L'optimisation de cette étape est cruciale pour aboutir à une sélectivité optimale mais également très délicate car les interactions analyte-polymère sont plus ou moins fortes en fonction de la nature de l'analyte et de la structure des cavités. En effet, toutes les cavités ne sont pas identiques. Ceci résulte de la structure meso- et macroporeuse des polymères [34]. De ce fait, l'optimisation de l'étape de lavage consiste à éliminer les interactions non spécifiques sans perdre les composés les plus faiblement retenus spécifiquement.

Pour vérifier la spécificité des interactions impliquées dans un processus d'extraction et éventuellement l'optimiser, l'approche couramment décrite consiste à comparer la rétention des analytes sur le MIP à celle sur un polymère non imprimé obtenu par la même voie de synthèse mais sans introduire la molécule empreinte. Dans le cas des composés très polaires, la réduction des interactions non spécifiques est difficile car ils interagissent fortement par liaisons hydrogène avec la matrice polymérique [37]. Une élimination totale de ces interactions parasites implique l'utilisation d'un solvant polaire protique ce qui peut entraîner également l'élution des composés les plus faiblement retenus spécifiquement [44].

Lorsque l'on cherche à appliquer directement un échantillon aqueux sur le MIP, il convient, pour développer

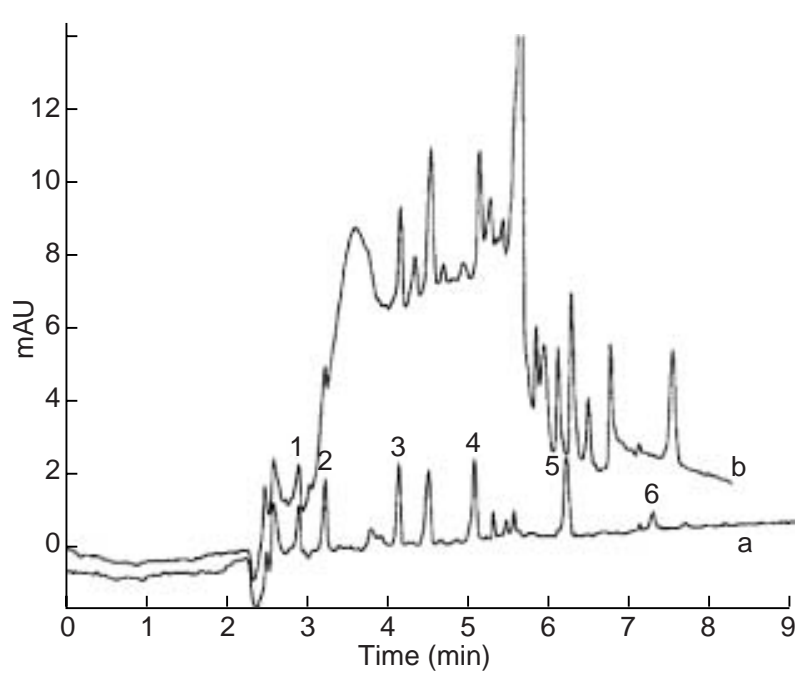

Figure 4

Analyse d'une eau de source dopée par des triazines et leurs métabolites $(100 \mathrm{ml}$ à $0.5 \mu \mathrm{g} / \mathrm{l})$ après extraction sur un polymère hydrophobe (PS-DVB) suivie (a) ou non (b) d'une étape de purification sur un MIP. Composés : 1 - déisopropylatrazine ; 2 - déséthylatrazine ; 3 - simazine ; 4 - atrazine ; 5 - propazine ; 6 - prométryne. D'après [41].

Analysis of groundwater spiked with triazines and their metabolites $(100 \mathrm{ml}$ at $0.5 \mu \mathrm{g} / \mathrm{l})$ via preconcentration on a hydrophobic polymer (PS-DVB) followed (a) or not (b) by a purification step on a MIP. Compounds: 1 - desisopropylatrazine; 2 - desethylatrazine; 3 - simazine; 4 - atrazine; 5 - propazine and 6 - prometryn. From [41].

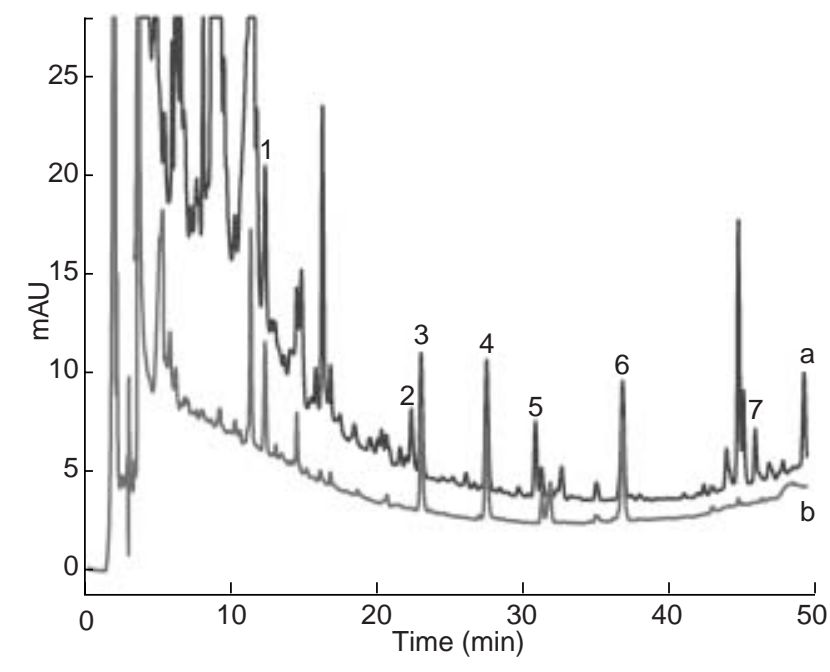

Figure 5

Chromatogrammes obtenus après extraction de $50 \mathrm{ml}$ d'un effluent industriel (dilué et dopé par des triazines et des phénylurées à $1 \mu \mathrm{g} / \mathrm{l}$ ) sur styrène divinylbenzène (a) et sur MIP terbutylazine (b). Triazines : 1 - dééthylatrazine ; 3 - déethylterbutylazine ; 4 - atrazine ; 6 - terbutylazine. Phénylurées : 2 - monuron ; 5 - diuron ; 7 - néburon. Détection UV à $220 \mathrm{~nm}$. D'après [37].

Chromatograms obtained after preconcentration of $50 \mathrm{ml}$ of an industrial effluent (diluted and spiked at $1 \mu \mathrm{g} / \mathrm{l}$ with triazines and phenylureas) on styrene-divinylbenzene (a) and on terbutylazine MIP (b). Triazines: 1 - deethylatrazine; 3 - deethylterbutylazine; 4 - atrazine; 6 - terbutylazine. Phenylureas: 2 - monuron; 5 - diuron; 7 - neburon. UV detection at $220 \mathrm{~nm}$. From [37]. 
une interaction sélective entre les analytes et les cavités spécifiques, d'introduire dans la procédure d'extraction, un solvant permettant de générer les interactions adéquates. Cependant, quelques travaux mentionnent des problèmes d'effets de matrice qui se traduisent par des baisses de rendement d'extraction lorsque les solutés sont recherchés dans des matrices réelles et non plus dans des eaux pures dopées [43, 45]. Pour un MIP fondé sur l'utilisation de méthacrylate comme monomère, nous avons mis en évidence des problèmes liés à la présence de cations dans les eaux naturelles (notamment $\mathrm{Ca}^{2+}$ ) dont l'effet est de bloquer, par échange d'ions, les sites d'interaction donneurs de liaisons hydrogène [37]. Au pH des eaux naturelles, les fonctions carboxyliques sont ionisées et ne peuvent plus développer des liaisons hydrogène avec les solutés. Ce problème a été résolu par l'introduction d'une étape de lavage à l'acide chlorhydrique après l'étape de percolation de l'échantillon. La solution acide permet d'éliminer les cations interagissant avec la matrice polymérique par échange d'ions avec les protons avant passage du solvant de lavage favorisant les interactions polaires (solvant peu polaire et aprotique). Cette méthode a été appliquée à l'extraction sélective de triazines d'un effluent industriel. Le résultat est illustré Figure 5. L'apport en sélectivité du MIP est démontré par le fait qu'il permet de limiter considérablement la présence d'interférents dans l'extrait analysé. De plus, l'application de la solution acide de régénération avant percolation de dichlorométhane (solvant de lavage) permet de supprimer les effets de matrice et d'obtenir, pour tous les échantillons d'eau analysés, des rendements d'extraction identiques à ceux obtenus pour l'eau pure dopée. L'apport en sélectivité visible en Figure 5 par la réduction du massif d'interférents est aussi démontré par le fait que des phénylurées, composés de même taille et appartenant à la même gamme de polarité que les triazines, n'ont pas été piégées par le MIP.

\section{QUELQUES EXEMPLES D'APPLICATION AUX PRODUITS PÉTROLIERS}

De nombreuses publications traitent de l'analyse des hydrocarbures polyaromatiques dans les eaux, sédiments sols, etc. suite à une pollution accidentelle par des produits pétroliers. Le cas des matrices aqueuses a été traité précédemment et une revue récente est consacrée à leur détermination dans les huiles et autres matrices graisseuses [46].

Les produits pétroliers sont des milieux extrêmement complexes et les applications de l'extraction en phase solide sur ces matrices sont surtout un enrichissement et fractionnement entre composés aliphatiques et aromatiques. En effet la séparation des pétroles en groupes hydrocarbonés est nécessaire en géochimie organique car un grand nombre de composés sont présents à l'état de traces et doivent être concentrés avant analyse par chromatographie en phase gazeuse (CPG) couplée à une détection par ionisation de flamme ou spectrométrie de masse. L'adsorbant souvent utilisé est la silice vierge et on peut trouver des exemples de fractionnement de carburants diesel en composés aliphatiques et hydrocarbures mono-, di- et poly-aromatiques en utilisant des microcolonnes en verre remplie de silice vierge [47]. Cependant, il faut activer la silice ce qui entraine d'ajuster exactement les quantités de traces d'eau présentes pour avoir des résultats reproductibles et d'autre part la sélectivité pour les alkylnaphthalenes est faible.

Bennet et Larter [48] ont décrit une procédure de SPE employant de la silice imprégnée avec du nitrate d'argent, ce qui permet une bonne séparation entre les hydrocarbure aromatiques et aliphatiques contenus dans un pétrole brut. Une première étape consiste en un isolement des hydrocarbures en percolant $100 \mathrm{mg}$ de pétrole de faible viscosité sur une cartouche remplie de silice greffée $\mathrm{C}_{18}$ (non traitée pour élimination des silanols résiduels) et en éluant avec $5 \mathrm{ml}$ d'hexane. Pour les pétroles de haute viscosité et contenant des hydrocarbures de haut poids moléculaires, le brut est dissous dans du chlorure de méthylène et un cartouche remplie de Florisil est préférée pour isoler les hydrocarbures totaux. Dans un second temps, l'extrait $(50 \mu \mathrm{l})$ de la cartouche $\mathrm{C}_{18}$ ou de celle de Florisil est fractionné par percolation sur une silice argentée $(550 \mathrm{mg}$ ) et les 2 premiers $\mathrm{ml}$ d'hexane contiennent les hydrocarbures aliphatiques alors que les $4 \mathrm{ml}$ suivants contiennent les aromatiques. La procédure a été testée avec plusieurs échantillons et à la fois la sélectivité et la reproductibilité ont été démontrées.

Un autre exemple plus récent a été développé pour la détermination rapide des empreintes suite au déversement accidentel issu du « Prestige ». Un fractionnement rapide et simple a été développé pour la quantification des fractions aliphatiques et aromatiques des résidus pétroliers avant caractérisation par GC-MS [49]. Une première cartouche a été remplie de silice vierge $\left(\mathrm{SiO}_{2}, 500 \mathrm{mg}\right)$, une seconde de silice greffée cyanopropyle $\left(\mathrm{C}_{3}-\mathrm{CN}, 500 \mathrm{mg}\right)$ et une troisième d'un mélange de $\mathrm{SiO}_{2}, 1000 \mathrm{mg}$ et $\mathrm{C}_{3}$-CN, $500 \mathrm{mg}$. Une élution a été réalisée par des fractions successives d'hexane, puis d'hexane contenant $20 \%$ de chlorure de méthylène. Un bon fractionnement entre aliphatiques $(92 \%$ dans $4,5 \mathrm{ml}$ d'hexane) et aromatiques (99\% dans les $4,5 \mathrm{ml}$ suivants contenant $20 \%$ de $\mathrm{CH}_{2} \mathrm{Cl}_{2}$ ) a été obtenu avec la troisième cartouche. Dans la première fraction on trouve la totalité des $n$-alcanes, isoprenoïdes, alkylcyclohexanes, hopanes, stéranes et diastéranes et dans la seconde, la totalité des polyaromatiques de deux à six cycles aromatiques, des alkyldithiobenzothiophènes et des stéroides triaromatiques. Seuls les alkylbenzènes à longue chaîne se retrouvent dans les deux fractions avec les deux tiers dans la première.

Les produits plus polaires sont plus faciles à concentrer par simple SPE sur silice vierge. Une application récente concerne l'identification des composés soufrés issus de la biodésulfuration des méthylbenzothiophènes contenus dans les pétroles fossiles après dégradation bactérienne [50]. Ces produits de dégradation sont concentrés par percolation sur une cartouche remplie de $500 \mathrm{mg}$ de silice vierge. Un lavage 
par $3 \mathrm{ml}$ d'hexane et $0,5 \mathrm{ml}$ d'éthylacétate-éthanol $(6 / 4, \mathrm{~V} / \mathrm{V})$ permet d'éliminer tous les composés apolaires et les composés recherché sont élués par $1 \mathrm{ml}$ d'éthylacétate-éthanol (6/4, V/V). L'analyse par GC-AED et GC-MS a permis l'identification des produits de désulfuration de quatre méthyldibenzothiophènes.

\section{CONCLUSION}

L'extraction en phase solide présente un fort potentiel pour l'extraction et la concentration de composés à partir de matrice liquides. La diversité des supports disponibles permet d'appliquer cette méthode à des composés de nature physicochimiques très variée. Le développement d'automate permet en plus de traiter en un temps limité un grand nombre d'échantillons. L'émergence de phases sélectives comme les immunoadsorbants et les MIP permet d'obtenir des extraits plus propres. Cela peut rendre alors la quantification finale plus fiable mais permettre aussi un gain en temps d'analyse pour laquelle il n'est alors plus nécessaire d'obtenir un grand pouvoir de séparation, principalement si la détection est réalisée par spectrométrie de masse.

Les produits pétroliers sont des matrices très complexes, mais quelques exemples illustrent bien l'utilité de la SPE pour concentrer et fractionner des familles de composés avant analyse.

\section{BIBLIOGRAPHIE}

1 Hennion, M.-C. (1999) J. Chromatogr., A 856, 3-54.

2 Hennion, M.-C. et Pichon, V. (1994) Environ. Sci. Technol., 28, 576A-583A.

3 Barcelo, D. et Hennion, M.-C. (1997) dans «Trace determination of pesticides and their degradation products in water», Barcelo, D. et Hennion M.-C.(eds), Elsevier, Amsterdam.

4 Poole, C.F., Gunatilleka, A.D. et Sethuraman, R. (2000) J. Chromatogr., A 885, 17-39.

5 Hennion, M.-C., Cau-dit-Coume, C. et Pichon, V. (1998) J. Chromatogr., A 823, 147-161.

6 Guenu, S. et Hennion, M.-C. (1994) J. Chromatogr., A 665 , 243-251.

7 Pichon, V., Cau-dit-Coume, C., Chen, L., Guenu, S. et Hennion, M.-C.(1996) J. Chromatogr., A 737, 25-33.

8 Masque, N., Galia, M., Marce, M. et Borull, F. (1997) Analyst, 122, 425-428.

9 D’Ascenzo, G., Gebtili, A., Marchese, S., Marino, A. et Perret D. (1998) Chromatographia, 48, 497-505.

10 Hennion, M.-C., Coquart, V., Guenu, S. et Sella, C. (1995) J. Chromatogr., A 712, 287-301.

11 Machtalère, G., Pichon, V. et Hennion, M.-C. (2000) J. High Resolut. Chromatogr., 23, 437-444.

12 Hennion, M.-C. (2000) J. Chromatogr., A 885, 73-95.

13 Guenu, S et Hennion, M.-C. (1997) J. Chromatogr., A 737, $15-23$.
14 Geerdink, R.B., Koosistra-Sijspersma, A., Tiesnitch, J., Kienhuis, P.G. et Brinkman, U.A.Th. (1999) J. Chromatogr., A 863, 147-155.

15 Pichon, V., Charpak, M. et Hennion, M.-C. (1998) J. Chromatogr., A 795, 83-92.

16 Boos, K.S. et Rudolphi, A. (1998) LC-GC Intern., 11, 45-54.

17 Boos, K.S. et Rudolphi, A. (1998) LC-GC Intern., 11, 84-95.

18 Delaunay, N., Pichon, V. et Hennion, M.-C. (2000) J. Chromatogr., B 745, 15-37.

19 Delaunay-Bertoncini, N., Pichon, V. et Hennion, M.-C. (2001) $L C-G C$, 14, 162-172.

20 Tsikas, D. (2001) J. Biochem. Biophys. Methods, 49, 705731.

21 Pichon, V., Delaunay-Bertoncini, N. et Hennion, M.-C (2002) Immunosorbents in sample preparation dans "Comprehensive Analytical Chemistry, Vol. XXXVII: Sampling and sample preparation for field and laboratory" Pawliszyn, J. (ed.), Elsevier, Chap. 33, pp 1081-1100.

22 Hennion, M.-C. et Pichon, V. (2003) J. Chromatogr., A 1000, 29-52.

23 Delaunay-Bertoncini, N., Pichon, V. et Hennion, M.-C. (2003) J. Chromatogr., A 999, 3-15.

24 Bouzige, M., Machtalère, G., Legeay, J., Pichon, V. et Hennion, M.-C. (1999) Waste Managment, 19, 171-180.

25 Bouzige, M., Legeay, P., Pichon, V. et Hennion, M.-C. (1999) J. Chromatogr., A 846, 317-329.

26 Pichon, V., Bouzige, M., Miège, C. et Hennion, M.-C. (1999) Trends in Analytical Chemistry, 18, 219-235.

27 Pichon, V., Aulard-Macler, E., Oubihi, H., Sassiat, P., Hennion, M.-C. et Caude, M. (1997) Chromatographia, 46, 529-538.

28 Bouzige, M., Pichon, V. et Hennion, M.-C. (1999) Environ. Sci. Technol., 33, 1916-1925.

29 Miège, C., Bouzige, M., Nicol, S., Dugay, J., Pichon, V. et Hennion M.-C. (1999) J. Chromatogr., A 859, 29-39.

30 Bouzige, M., Pichon, V. et Hennion, M.-C. (1998) J. Chromatogr., A 823, 197-210.

31 Schoenzetter, E., Pichon, V., Thiebaut, D., Fernandez-Alba, A. et Hennion, M.-C. (2000) J. Microcolumn Separations, 12, 316-322.

32 Pichon, V., Chen, L., Durand, N., Le Goffic, F. et Hennion, M.-C. (1996) J. Chromatogr., A 725, 107-119.

33 Pichon, V., Rogniaux, H., Fisher-Durand, N., Ben Rejeb, S., Le Goffic, F. et Hennion, M.-C. (1997) Chromatographia, 45, 289-295.

34 Sellergren, B. et Lanza, F. (2001) «Molecularly imprinted polymers in solid phase extractions» chap. 15 dans «Molecularly imprinted polymers - man-made mimics of antibodies and their applications in analytical chemistry», Sellergren, B. (ed) (Vol. 23 de la série "Techniques and Instrumentation in Analytical Chemistry »), Elsevier, 355375.

35 Lanza, F. et Sellergren, B. (2001) Chromatographia, 53, 599611.

36 Ramström, O., Skudar, C., Haines, J., Patel, P. et Brüggemann, O. (2001) J. Agric. Food Chem., 49, 21052114.

37 Chapuis, F., Pichon, V., Lanza, F., Sellergren, B. et Hennion, M.-C. (2003) J. Chromatogr., A 999, 23-33.

38 Zander, A., Findlay, P., Renner, T. et Sellergren, B. (1998) Anal. Chem., 70, 3304-3314. 
39 Martin, P., Wilson, I.D. et Jones, G.R. (2000) Chromatographia, 52, S19-S23.

40 Baggiani, C., Giovanolli, C., Anfossi, L. et Tozzi, C., (2001) J. Chromatogr., A 938, 35-44.

41 Turiel, E., Martin-Esteban, A., Fernandez, P., Perez-Conde, C. et Camara, C. (2001) Anal. Chem., 73, 5133-5144.

42 Koeber, R., Fleischer, C., Lanza, F., Boos, K.-S., Sellergren, B. et Barcelo, D. (2001) Anal. Chem., 73, 2437-2444.

43 Pap, T., Horvath, V., Tolokan, A., Horvai, G. et Sellergren, B. (2002) J. Chromatogr., A 973, 1-12.

44 Baggiani, C., Giovanolli, C., Anfossi, L. et Tozzi C. (2001) J. Chromatogr., A 938, 35-44.

45 Masqué, N., Marcé, R.M., Borrull, F., Cormack, P.A.G. et Sherrington, D.C. (2000) Anal. Chem., 72, 4122-4126.
46 Moret, S. et Conte, L.S. (2000) J. Chromatogr., A 882, 245253.

47 Bundt, J., Herbel, W., Steinhart, H., Franke, S. et Franke, W. (1991) J. High Resolut. Chromatogr., 14, 91-98.

48 Bennet, B. et Larter, S.R. (2000) Anal. Chem., 72, 10391044.

49 Alzaga, R., Montuori, P., Ortiz, L., Bayona, J. et Albaiges J. (2004) J. Chromatogr., A 1025, 133-138.

50 Onaka, T., Morio Kobayashi, M., Yoshitaka Ishii, Y., Koichi Okumura, K., et Masanori Suzuki, M. (2000) J. Chromatogr., A 903, 193-202. 